\title{
Perioperative Stroke and Thirty-Day Hospital Readmission After Cardiac Surgeries: State Inpatient Database Study
}

\author{
Nada Alrifai ${ }^{\mathrm{a}, \mathrm{e}}$, Laith Alhuneafat ${ }^{\mathrm{a}, \mathrm{e}}$, Khaled AlRobaidi ${ }^{\mathrm{b}}$, Samir S. Al Ghazawic, \\ Parthasarathy D. Thirumala ${ }^{\mathrm{d}, \mathrm{f}}$
}

\begin{abstract}
Background: Readmission rates are an important metric for evaluating healthcare quality. Stroke is a major complication following cardiac surgery. Our study aimed to evaluate the frequency and predictors of 30-day unplanned hospital readmission after cardiac surgeries and to evaluate the impact of perioperative stroke on readmission.

Methods: Surgical discharge records spanning the years of 2008 through 2011 were analyzed utilizing California State Inpatient Database. International Classification of Diseases, ninth revision-Clinical Modification (ICD-9-CM) codes and Clinical Classification Software (CCS) codes were used to identify surgeries and variables of interest. Surgical records were then followed up for 30 days through linking admission records. Perioperative stroke was defined as brain infarction of ischemic or hemorrhagic etiology that occurred during or within 30 days after surgery.

Results: Baseline characteristics associated with increased readmission rates were female gender, age above 65 , non-white race, lower income, and increased number of comorbidities. Among 199,617 hospitalizations for cardiac surgeries, 1,817 (0.91\%) patients developed perioperative stroke. The rate of readmission in perioperative stroke patients was $21.89 \%$. They had a longer length of hospital stay and their discharge was vastly non-routine (84\%). Our univariate analysis yielded significant association between stroke and readmission rates (odds ratio: 1.82, 95\% confidence interval: 1.63 - 2.04). This association failed to remain significant upon controlling for other variables in our multivariate analysis.
\end{abstract}

Manuscript submitted December 1, 2021, accepted December 23, 2021

Published online January 29, 2022

aDepartment of Internal Medicine, Allegheny Health Network, Pittsburgh, PA, USA

bepartment of Neurology, University of Alabama, Birmingham, AL, USA

'Department of Neurology, Memorial Healthcare System, Hollywood, FL, USA

dDepartment of Neurological Surgery, University of Pittsburgh, Pittsburgh, PA, USA

'These authors contributed equally to this article.

${ }^{f}$ Corresponding Author: Parthasarathy D. Thirumala, Department of Neurological Surgery, UPMC Presbyterian Hospital, Pittsburgh, PA 15213, USA. Email: thirumalapd@upmc.edu

doi: https://doi.org/10.14740/jocmr4647
Conclusion: Baseline patient characteristics and perioperative complications are significant predictors of readmission. More than one in five patients who develop a stroke after cardiac surgery are readmitted to the hospital within 30 days of discharge.

Keywords: 30-day Readmission; Perioperative stroke; Cardiac procedure; Readmission; Cardiac surgery; Stroke

\section{Introduction}

Reducing hospital readmissions is a national priority aimed to improve the quality of care and reduce unnecessary health cost [1,2]. A study by Lawson et al [3] established a link between the occurrence of postoperative complications and the risk of readmission. Results demonstrated almost a fourfold increased risk of readmission for patients who have one or more postoperative complications. The study also revealed that moderate reductions in complication rates of $5-20 \%$ corresponded with a decrease in readmissions and expense savings for Medicare ranging from $\$ 31$ million to $\$ 124$ million per year. Cardiac surgery is an intriguing topic in this regard as it has high shortterm readmission rates $(16.5 \%)[4,5]$.

Factors that determine risk of readmissions vary. Other than the preoperative condition of patients which encompass their age, gender, and presence of comorbidities, postoperative complications also have a highly significant impact on readmission rates. Perioperative stroke is a postoperative complication of substantial interest following cardiovascular surgeries with an incidence ranging from $1.9 \%$ to $9.7 \%$ [6] and the majority of strokes occurring within 7 days $[7,8]$. This rate is relatively high compared to an incidence of approximately $0.9-1.9 \%$ in noncardiac, non-neurologic, and non-major surgery [9].

It has been presented that perioperative strokes lengthen inhospital stays, leading to disabilities and discharge to long-term care facilities [10-12]. However, not enough data exist about readmission rates following perioperative strokes after cardiovascular surgeries. Investigations must be made as hospitals are frequently penalized for having high readmission rate [13]. A closer look at readmissions during the 30-day period might provide us with a better insight on how perioperative stroke affects hospital outcomes. The fact that hospital readmissions may be reduced, given that they are potentially preventable, makes it a proposed way to improve quality of care [14]. 
Our primary aim in this study was to determine predictors of readmission within the first 30 days after cardiac surgery. Our secondary aim was to evaluate the impact of perioperative stroke following cardiac surgeries on the frequency of readmissions.

\section{Materials and Methods}

\section{Data source}

A study population of adults undergoing cardiac surgeries was identified using the State Inpatient Database (SID). Data were abstracted from the California SID for the years 2008 to 2011. CA-SID encompasses about $97 \%$ of California state community hospital inpatient discharge records, regardless of payer type. It has more than 100 clinical and non-clinical variables including diagnoses (primary, secondary), list of surgeries performed, admission status, discharge status, demographics, length of stay and comorbidities. The reason our study was conducted starting the year 2008 was due to introduction of the present on admission (POA) variable in 2008. SID is unique in terms of presenting admission variables which differentiate whether a patients' diagnosis was identified prior to admission or as a complication during hospitalization. International Classification of Diseases, ninth revision-Clinical Modification (ICD-9-CM) codes and Clinical Classification Software (CCS) codes were used to identify surgeries and isolate variables of interest. The SID data used in our analyses are available for purchase from the US Agency for Healthcare Research and Quality (AHRQ) [15]. The study was granted ethical exempt status from the University of Pittsburgh IRB.

\section{Study population}

This study included patients of age greater than 18 who underwent cardiac surgeries during the years 2008 through 2011. The reason for omitting younger age groups was due to the relatively lower risk of developing perioperative stroke [16]. A total of 199,617 unique patients met inclusion criteria for the study. The ICD-9-CM coding system was utilized for identification of major cardiac procedures that patients underwent during the index hospital admission according to the CCS for surgical procedures. Surgical procedures done for diagnostic purposes and non-operating room procedures were excluded, secondary to relatively lower rates of stroke. Patients who died within 30 days of their surgery or had scheduled/planned readmission for any purpose, including rehabilitation, were also excluded. The surgeries included were: heart valve procedures, coronary artery bypass graft $(\mathrm{CABG})$, insertion; revision; replacement; removal of cardiac pacemaker or cardioverter/defibrillator, other operating room heart procedures, and extracorporeal circulation auxiliary to open heart procedures.

\section{Patient characteristics, hospital readmission and outcomes}

Patient demographics covariates included age, sex, race, pri- mary payer and median income levels. Clinical covariates included admission route (i.e., routine or non-routine); Elixhauser comorbidity variables (hypertension, diabetes, etc.); van Walraven score groups (VWS); and discharge location (routine, skilled nursing facility (SNF), etc.). For the purpose of our study, perioperative stroke is defined as a brain infarction of ischemic or hemorrhagic etiology that occurs during surgery or within 30 days after surgery. Thirty-day hospital readmissions were determined based on a definition delineated by Healthcare Cost and Utilization Project (HCUP). Primary ICD-9 diagnosis codes were used to determine indication for hospital readmissions.

\section{Statistical analysis}

Dataset construction and statistical analyses were performed using SAS 9.4. CCS codes available on HCUP website were used to group surgical procedures into different categories, while ICD-9 codes were used to search for selected surgeries that may be associated with an incidence of perioperative stroke (Supplementary Material 1, www.jocmr.org). Continuous variables were outlined as mean \pm standard deviation (SD). Categorical values were reported as percentages. We ran an initial univariate analysis using Chi-square for categorical variables and $t$-tests for continuous variables. After testing for multicollinearity, we ran several logistic models to calculate adjusted Wald odds ratios (ORs) for predictors of readmission. Our predictor variables included age, gender, race/ethnicity, household median income, and usage of cardiopulmonary bypass (CPB) machine during cardiac surgery, admission source, discharge location, and complications. The primary study outcome was 30-day hospital readmission.

\section{Results}

\section{Patient characteristics}

A total of 199,617 patients with a mean age of $69 \pm 13.92$ years, who underwent major cardiac surgeries, in California from 2008 through 2011, were identified from the SID. Our study population included $62.67 \%$ males and $37.33 \%$ females. Most patients included in our study were white constituting (68.33\%) followed by Hispanic race (15.63\%), Asian (8.28\%), African American (5.15\%) and others (2.6\%). Majority of patients included in our study were of low-risk category with $70.33 \%$ falling under a risk group category of $<5$ on the VWS scale. Medicare insurance encompassed $62.03 \%$ of patients, the private insurance covered $26.48 \%$ of patients, and Medicaid paid for $6.93 \%$ of overall patients. An evenly distributed number of patients were within the range of each of the household income quartiles: first quartile $23.15 \%$, second quartile $24.75 \%$, third quartile $26.23 \%$, and fourth quartile $25.87 \%$. The frequency of cardiac surgeries performed is as follows: heart valve procedures $17.03 \%(\mathrm{n}=33,997)$, CABG $23.08 \%$ $(\mathrm{n}=46,076)$, cardiac pacemaker and defibrillator related procedures $44.46 \%(\mathrm{n}=88,750)$ and other operation room related 
heart procedure $15.36 \%(\mathrm{n}=30,655)$ (Supplementary Material 2, www.jocmr.org). Patients who were put on CPB machine during major cardiac surgeries constituted $37.12 \%(\mathrm{n}=$ 74,098) of overall patients.

\section{Perioperative stroke}

Among the 199,617 invasive cardiac surgeries performed, $1,809(0.91 \%)$ patients developed perioperative stroke during index hospitalization. Baseline characteristics of patients with and without perioperative strokes are demonstrated in Table 1. Compared to patients who did not develop stroke, patients with perioperative stroke were older $(71.53 \%$ vs. $65.7 \%$ above the age of 65 ), more likely to be female (40.6\% vs. $37.3 \%$ ) and none white $(34.23 \%$ vs. $31.64 \%)$, had a higher comorbidity burden (69.1\% vs. $29.32 \%$ with VWS $>5)$, were more frequently put on $\mathrm{CPB}$ machine during surgery $(70.76 \%$ vs. $36.81 \%)$, and had a significantly longer length of stay $(19.7 \pm$ 19.6 SD vs. $6.7 \pm 7.3$ SD). Majority of patients were routinely admitted for their surgeries. However, patients who developed perioperative stroke were more likely to be discharged to SNFs, intermediate care facilities (ICFs), and other healthcare equivalents including home healthcare and transfer to other short-term hospitals compared to patients who did not develop perioperative stroke ( $84.28 \%$ vs. $36.43 \%)$.

Majority of the patients who had perioperative stroke had insurance coverage through Medicare $(\mathrm{n}=1,182,65.34 \%)$ with fewer patients covered by private insurance $(\mathrm{n}=398$, $22 \%)$, Medicaid $(n=149,8.24 \%)$, and other sources $(4.42 \%)$. Comorbidities highly associated with development of perioperative stroke were paralysis, other neurological disorders, peptic ulcer disease, weight loss, valvular disease and congestive heart failure (Table 1). The CPB machine was a recognized risk factor for developing a perioperative stroke with around fourfold increased incidence among patients who were placed on them during their surgery (CI: $3.75-4.60, \mathrm{P}<0.0001)$.

\section{0-day hospital readmission}

\section{Univariate analysis}

Overall, 26,751 (13.40\%) patients with a mean age of $70.4 \pm$ 13.7 were readmitted within 30 days following their invasive cardiac surgeries. The overall incidence of 30-day hospital readmission among all survivors of perioperative stroke was $21.89 \%$ (396 readmissions).

Baseline characteristics of patients readmitted within 30 days and their univariate analysis are presented in Tables 2 and 3. Patients readmitted within 30 days were more likely to be females than males (OR: 1.23, 95\% CI: 1.20 - 1.26), commonly older than 85 years old (OR: $1.34,95 \%$ CI: 1.29 - 1.38), of African American race (OR: 1.44, CI: 1.36 - 1.52), and of lower household incomes (first quartile: OR: 1.25, 95\% CI: 1.2 - 1.3). Emergent admissions at index hospitalization predicted an increased odds of readmission (OR: 1.56, 95\% CI: 1.52 - 1.61) after patient's discharge. Comorbidities including congestive heart failure, weight loss, pulmonary circulation disorders, renal failure, and solid tumor with metastases were most commonly associated with an increased incidence of readmission within 30 days. In general, patients with high comorbidity burden (VWS > 5) were more likely to be readmitted and incidence was highest among patients with VWS $>14$ (OR: 2.68, 95\% CI: 2.54 - 2.85). Cases where CPB machine was used during surgery had higher odds of 30-day readmission (OR: 1.26, 95\% CI: 1.23 - 1.30). The five most common complications associated with an increased readmission rate following invasive cardiac surgeries were in order wound, gastrointestinal, renal, infection and stroke (Tables 2 and 3). Non-routinely discharged patients to SNFs, ICFs, or other type of care facilities had highest incidence of readmission (OR: 2.61, 95\% CI: 2.52 - 2.7) followed by patients discharged to short-term hospitals or against medical advice (OR: 2.04, 95\% CI: 1.88 - 2.21) and with home healthcare (OR: 1.65, 1.59 - 1.7). Patients who were not readmitted were more likely to be white, admitted and discharged routinely, and had a higher household income.

\section{Multivariate logistic regression analysis}

In our logistic regression analysis, we found an association between certain patient demographics and increased rate of 30day readmissions. Patients who were female and of an age $>85$ were more likely to be readmitted (OR: $1.15,95 \%$ CI: 1.12 1.19 and OR: 1.06, 95\% CI: 1.02 - 1.10, respectively). Overall, non-white patients had higher odds of being readmitted. Out of all race groups, the African American race had the highest risk of being readmitted (OR: 1.33, 95\% CI: 1.25 - 1.41). Patients with a low socioeconomic status households had the highest risk of being readmitted (OR: 1.16, 95\% CI: $1.11-1.20)$.

A higher comorbidity burden was associated with higher odds of readmissions. Patients with the highest measure of comorbidity burden, VWS $>14$, had an OR of $1.85,95 \%$ CI of 1.73 - 1.97 compared to those with an intermediate comorbidity burden, VWS 5 - 14, having an OR of $1.43,95 \%$ CI of 1.38 - 1.47. CPB machine use during surgery was also associated with higher odds of readmission (OR: 1.20, 95\% CI: 1.16 1.24).

Admission source and discharge location played a significant impact on the risk of 30-day readmission following surgeries. Patients with non-routine admissions generally and emergent admissions specifically were at higher odds of being readmitted (OR: 1.40, 95\% CI: 1.36 - 1.44). Similarly, patients who had a non-routine discharge had higher odds of readmissions. Patients discharged to SNFs, ICFs, or other type of facilities had the highest risk of being readmitted within 30 days (OR: 1.93, 95\% CI: 1.86 - 2.01), followed with patients discharged to short-term hospitals or against medical advice (OR: 1.56, 95\% CI: 1.43 - 1.69) and being discharged with home healthcare (OR: 1.42, 95\% CI: 1.37 - 1.47).

Postoperative complications including renal, gastrointestinal, infection, respiratory and cardiovascular complications were associated with higher odds of 30-day readmissions. However, stroke as a postoperative complication was not associated with increased risk of readmission within 30 days (OR: 0.96, 95\% CI: 0.85 - 1.08). 
Table 1. Patient Characteristics With and Without Perioperative Stroke Following Cardiac Surgery

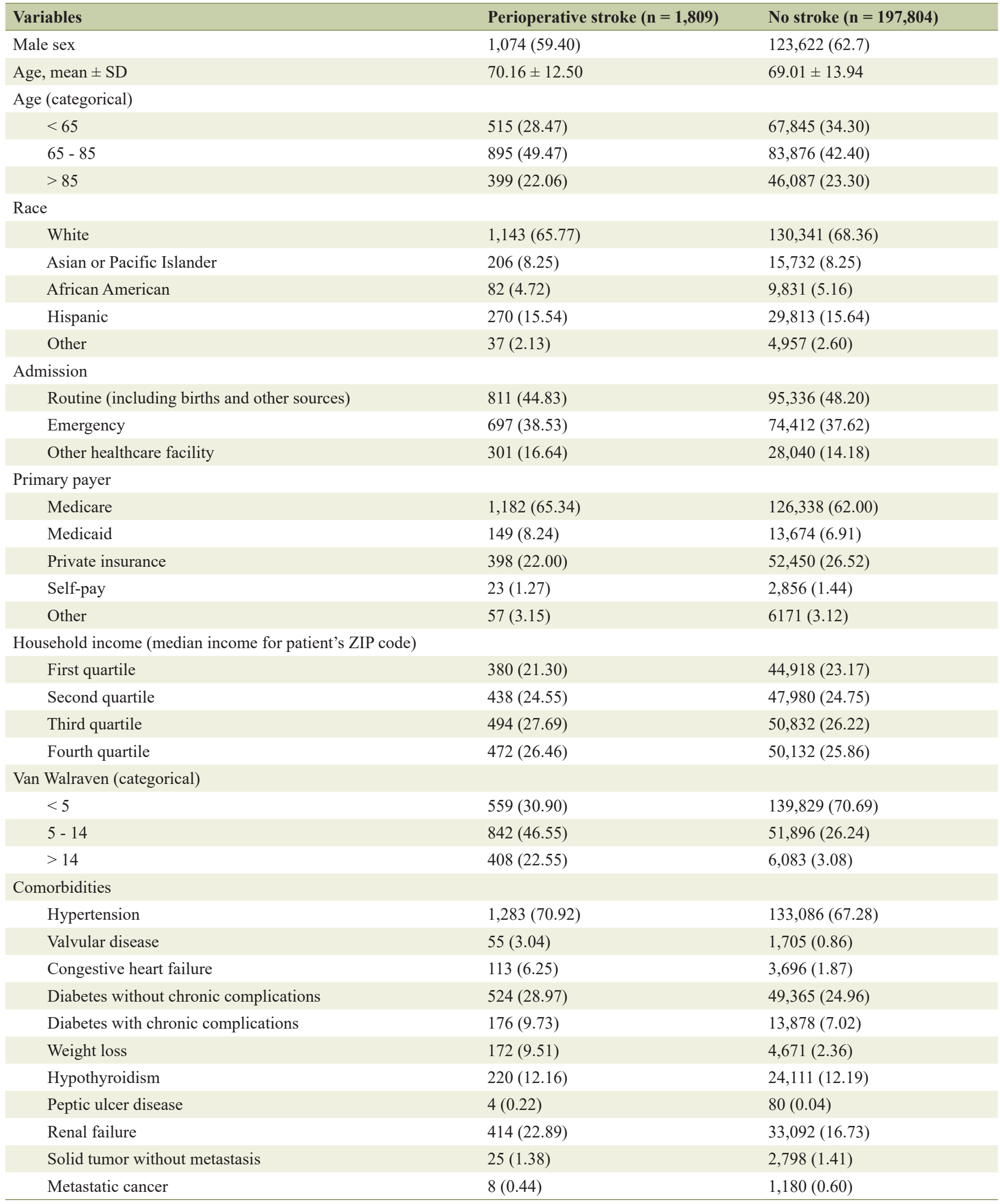


Table 1. Patient Characteristics With and Without Perioperative Stroke Following Cardiac Surgery - (continued)

\begin{tabular}{|c|c|c|}
\hline Variables & Perioperative stroke $(\mathrm{n}=\mathbf{1 , 8 0 9})$ & No stroke $(n=197,804)$ \\
\hline Lymphoma & $15(0.83)$ & $1,114(0.56)$ \\
\hline Pulmonary circulation disorders & $32(1.77)$ & $892(0.45)$ \\
\hline Liver disease & $39(2.16)$ & $3,533(1.79)$ \\
\hline Peripheral vascular disorders & $379(20.95)$ & $21,292(10.76)$ \\
\hline Paralysis & $670(37.04)$ & $3,222(1.63)$ \\
\hline Other neurological disorders & $471(26.04)$ & $9,492(4.80)$ \\
\hline Obesity & $246(13.60)$ & $27,548(13.93)$ \\
\hline Rheumatoid arthritis/collagen vascular diseases & $40(2.21)$ & $4,171(2.11)$ \\
\hline Chronic blood loss anemia & $45(2.49)$ & $2,629(1.33)$ \\
\hline Deficiency anemia & $575(31.79)$ & $41,946(21.21)$ \\
\hline Acquired immune deficiency syndrome & $3(0.17)$ & $272(0.14)$ \\
\hline Alcohol abuse & $65(3.59)$ & $5,247(2.65)$ \\
\hline Cardiopulmonary bypass machine & $1,280(70.76)$ & $72,818(36.81)$ \\
\hline \multicolumn{3}{|l|}{ Discharge } \\
\hline Routine & $284(15.72)$ & $125,742(63.57)$ \\
\hline $\begin{array}{l}\text { Transfer to short-term hospital or } \\
\text { discharged against medical advice }\end{array}$ & $155(8.58)$ & $3,838(1.94)$ \\
\hline
\end{tabular}

SD: standard deviation; SNF: skilled nursing facility; ICF: intermediate care facility.

Table 2. Patient Characteristics of Readmitted vs. Not Readmitted Within 30 Days After Cardiac Surgeries

\begin{tabular}{|lll}
\hline & Readmission $(\mathbf{n}=\mathbf{2 6 , 7 5 1})$ & No readmission $(\mathbf{n}=\mathbf{1 7 2 , 8 6 6 )}$ \\
\hline Female & $11,108(41.55)$ & $63,158(36.67)$ \\
\hline $\begin{array}{l}\text { Age, mean } \pm \text { SD } \\
\text { Age (categorical) }\end{array}$ & $70.4 \pm 13.7$ & $68.8 \pm 13.9$ \\
$\quad<65$ & $8,168(30.53)$ & $60,192(34.82)$ \\
$\quad 65-85$ & $11,441(42.77)$ & $73,330(42.42)$ \\
$\quad>85$ & $7,142(26.70)$ & $39,344(22.76)$ \\
Race & & $114,713(68.96)$ \\
$\quad$ White & $16,711(64.32)$ & $13,602(8.18)$ \\
$\quad$ Asian or Pacific Islander & $2,336(8.96)$ & $8,194(4.93)$ \\
$\quad$ African American & $1,719(6.59)$ & $25,581(15.38)$ \\
$\quad$ Hispanic & $4,502(17.27)$ & $4,249(2.55)$ \\
Other & $745(2.86)$ & $85,628(49.54)$ \\
\hline
\end{tabular}


Table 2. Patient Characteristics of Readmitted vs. Not Readmitted Within 30 Days After Cardiac Surgeries - (continued)

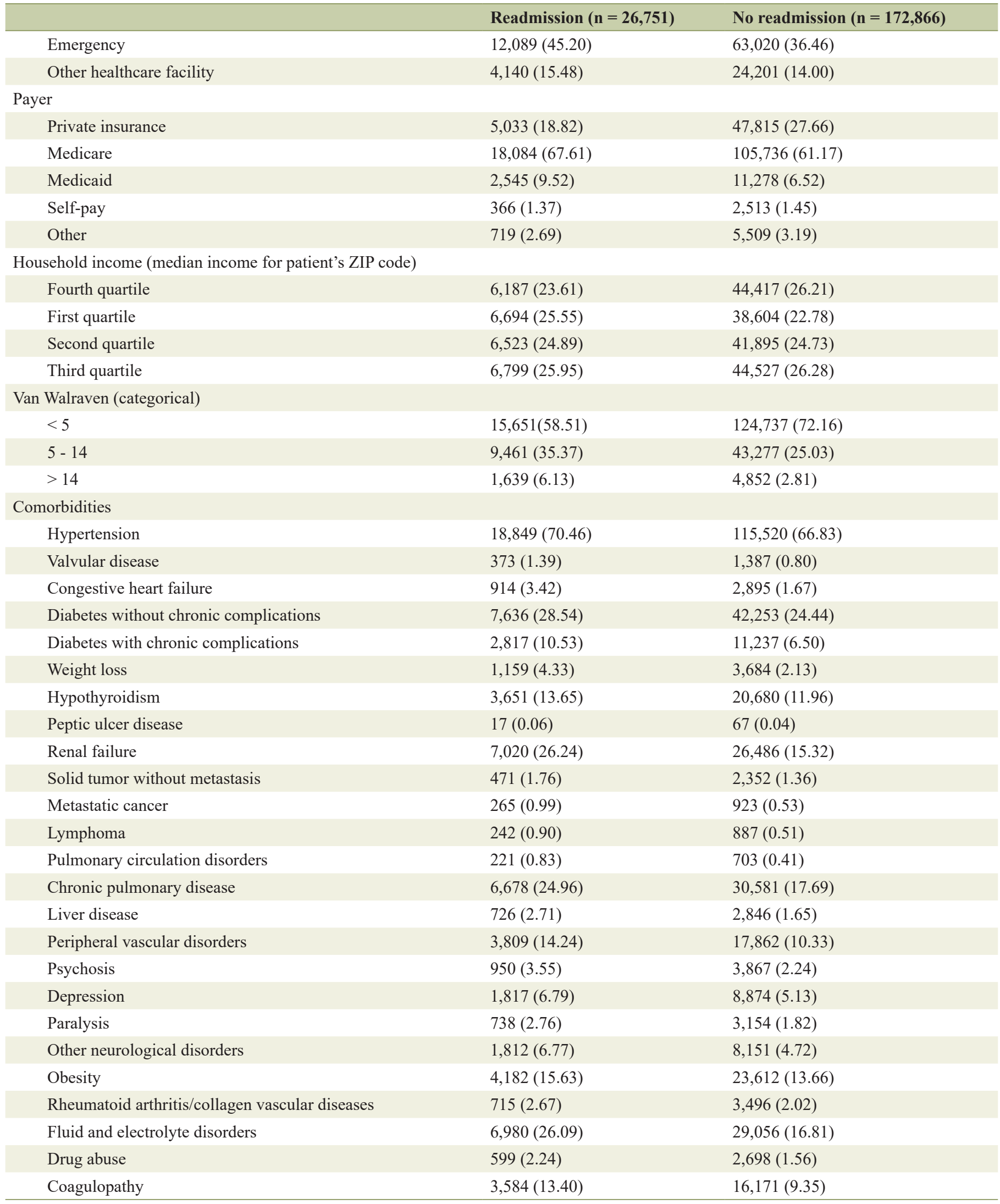


Table 2. Patient Characteristics of Readmitted vs. Not Readmitted Within 30 Days After Cardiac Surgeries - (continued)

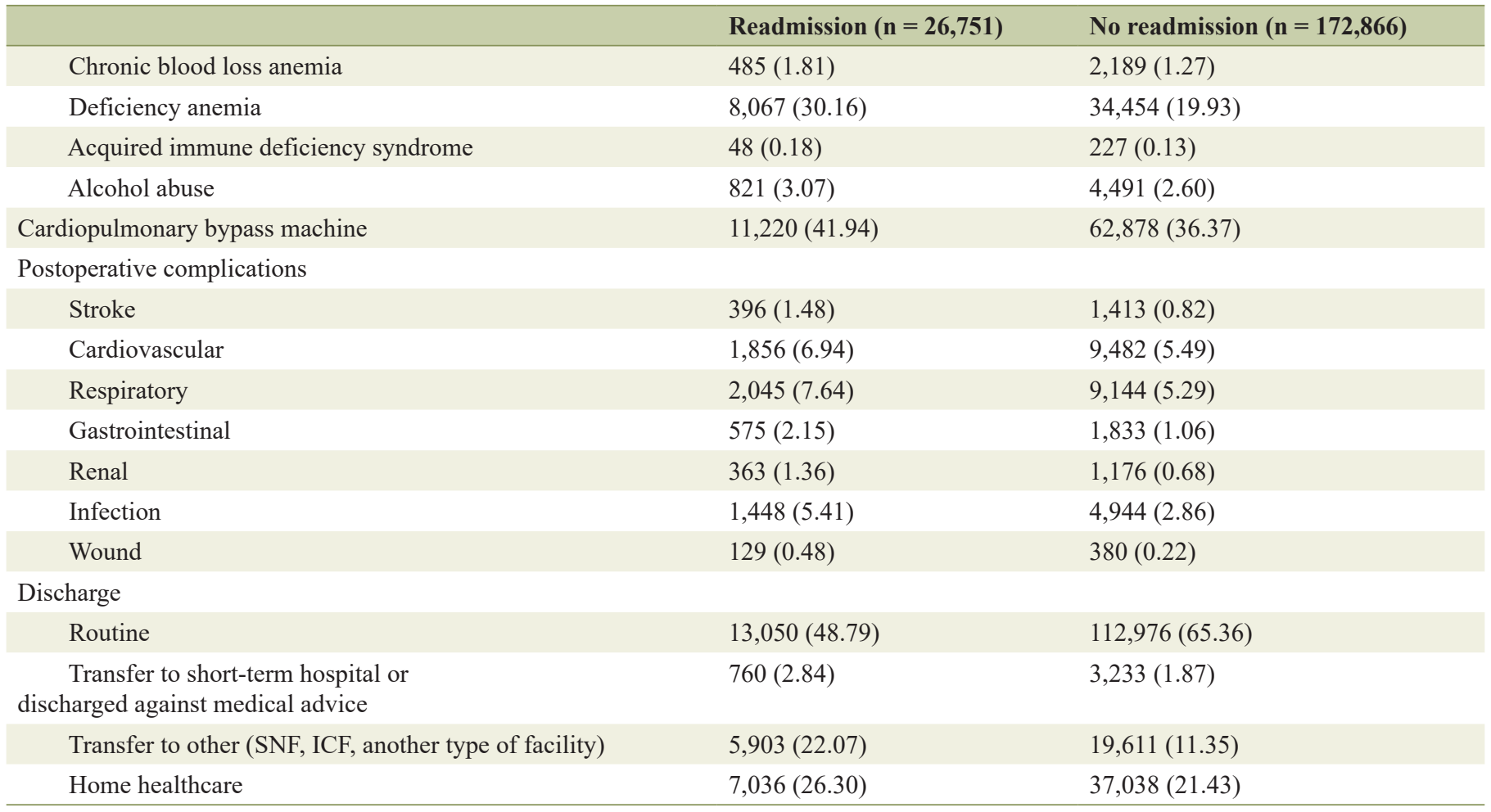

SD: standard deviation; SNF: skilled nursing facility; ICF: intermediate care facility.

Table 3. Odds Ratios Obtained by Unadjusted Univariate and Adjusted Multivariate Logistic Regression Analysis for 30-Day Readmission

\begin{tabular}{|c|c|c|c|c|}
\hline & Unadjusted odds ratio & $95 \%$ CI & Adjusted odds ratio & $95 \%$ CI \\
\hline Male & Ref & & Ref & \\
\hline Female & $1.23 *$ & $1.20-1.26$ & $1.15^{*}$ & $1.12-1.19$ \\
\hline$<65$ & Ref & & Ref & \\
\hline $65-85$ & $1.15^{*}$ & $1.12-1.19$ & 1.02 & $0.99-1.05$ \\
\hline \multicolumn{5}{|l|}{ Race } \\
\hline White & Ref & & Ref & \\
\hline Asian or Pacific Islander & $1.18^{*}$ & $1.12-1.23$ & $1.11 *$ & $1.06-1.17$ \\
\hline African American & $1.44^{*}$ & $1.36-1.52$ & $1.33 *$ & $1.25-1.41$ \\
\hline Hispanic & $1.2^{*}$ & $1.16-1.25$ & $1.16^{*}$ & $1.12-1.21$ \\
\hline Emergency & $1.56^{*}$ & $1.52-1.61$ & $1.40^{*}$ & $1.36-1.44$ \\
\hline Other healthcare facility & $1.39 *$ & $1.34-1.45$ & $1.26^{*}$ & $1.21-1.31$ \\
\hline \multicolumn{5}{|c|}{ Household income (median income for patient's ZIP code) } \\
\hline Fourth quartile & Ref & & Ref & \\
\hline First quartile & $1.25^{*}$ & $1.20-1.30$ & $1.16^{*}$ & $1.11-1.20$ \\
\hline
\end{tabular}


Table 3. Odds Ratios Obtained by Unadjusted Univariate and Adjusted Multivariate Logistic Regression Analysis for 30-Day Readmission - (continued)

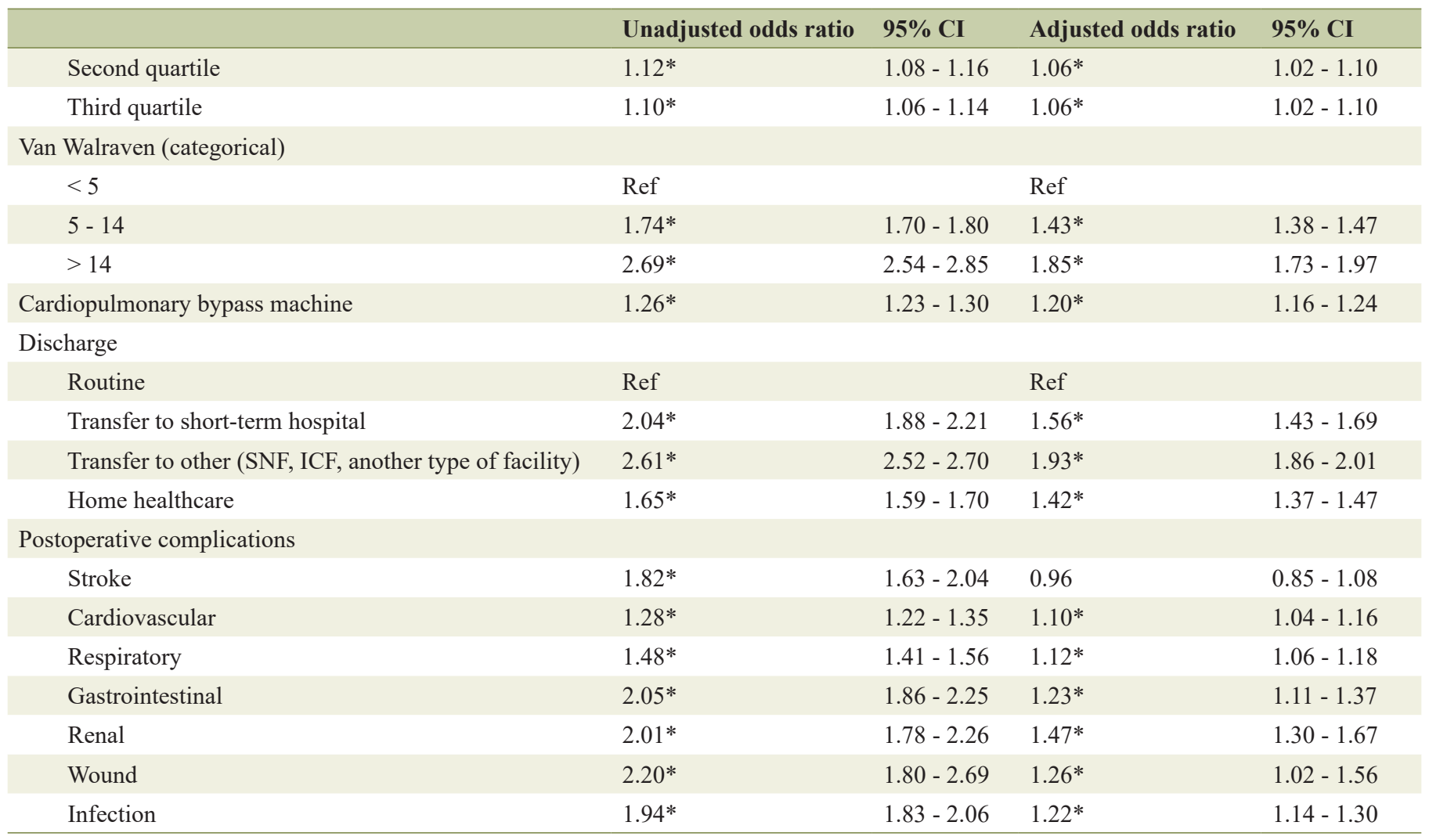

${ }^{*} \mathrm{P}<0.05 . \mathrm{Cl}$ : confidence interval; Ref: reference; SNF: skilled nursing facility; ICF: intermediate care facility.

\section{Discussion}

This is an SID study, looking at 30-day readmissions following cardiac surgeries. The study highlights characteristics and rates of readmission in patients who undergo cardiac surgeries and sheds light on patients who develop perioperative stroke in a setting of cardiac surgeries. Patients who undergo cardiac surgeries are at an increased risk of being readmitted if they have the following characteristics: female gender, age above 65 , none white race, lower household incomes and increased comorbidities. The rate of readmission in patients with perioperative stroke was one in five. Patients with perioperative stroke are more likely to be readmitted than those without perioperative stroke within a 30-day period (OR: 1.8 95\% CI: 1.63 - 2.04). When readmission was adjusted for other variables in our logistic regression analysis, several patient characteristics and perioperative complications predicted 30-day readmissions after cardiac surgeries; however, perioperative stroke is not amongst them (Table 3). To our knowledge and in widely searched literature, our study was the first to look at the impact of developing perioperative stroke on hospital health quality outcomes in terms of 30-day readmission following major cardiac surgeries.

Perioperative stroke is of high incidence among patients who undergo cardiac surgeries ranging from $1.6 \%$ to $9.7 \%$ [6, $17,18,19]$. In our study, several patient demographics are as- sociated with increased readmissions following cardiac surgeries. These findings are consistent with other studies looking at $\mathrm{CABG}$ surgeries and risk factors for 30-day readmissions, revealing the importance of patient baseline characteristics in predicting readmissions $[20,21]$. Other previous work addressed the link between lower socioeconomic status and increased risk of readmission [22, 23].

CPB machine is associated with an increased risk of perioperative stroke consistent with previous literature [24-26]. Precipitation of microemboli following the use of CPB machine during surgery implicates its use in developing perioperative stroke [27]. Our study also shows increased risk of readmission in patients who were on-pump during surgery consistent with previous studies [28, 29].

Although our multivariate analysis suggests that stroke as a postoperative complication is not associated with increased readmissions, other perioperative complications such as cardiovascular, respiratory, gastrointestinal, renal and infectious complications did. In a prospective study, evaluating causes of readmission after major cardiac surgery and other studies among patients undergoing CABG procedure, cardiovascular, respiratory, and infectious complications are found to be the most common causes of readmission within 30 days [20, 21, $30]$. The divergence between our multivariate and our univariate analysis concerning perioperative stroke being a risk factor for 30-day readmission may highlight the fact that although 
perioperative stroke does not directly lead to increased 30-day readmissions, the associated patient characteristics and comorbidities may put the patient at increased risk of 30-day readmission. Furthermore, perioperative stroke may lead to other complications, some of which may be potentially preventable, including pneumonia and urinary tract infections which are recognized by Centers for Medicare and Medicaid Services (CMS) and previous studies as predictors for readmission [13, 31-36].

For stroke survivors, longer lengths of stay at the hospital, significant disabilities, and discharge to rehabilitation facilities to recuperate are common outcomes. In our study, patients who develop perioperative stroke are more likely to be discharged to SNFs, ICFs, and other care facilities (Table 1). This may be explained by the rise of managed care plans where the incentives to providers/payers forced hospitals to reduce costs. Thus, patients who are viewed as acute rehabilitation are reevaluated to be treated in a nonhospital setting [37]. It is worthy of note that patients who are discharged to these facilities have the highest risk of being readmitted (OR: 1.93, 95\% CI: $1.855-2.007)$, compared to other routes of discharge. This may be explained by the implementation of Medicare Prospective Payment System. In attempt to cut down costs in provision of care to patients, hospitals aim to decrease the length of stay and discharge patients to other care facilities. However, these shifts in transfers to subacute settings have still not proven to meet the standards of care required for these patients. Furthermore, our data precede October 2018 before CMS issued a final rule supporting the shift of SNFs from fee-for-service to Value-Based Purchasing Programs. New trends may be seen in readmission rates from SNFs as Medicare adjusts reimbursements to SNFs based on their performance on the program's hospital readmissions measure [13].

Overall, stroke is among the 10 highest contributors to Medicare and Medicaid costs [38]. Hospital Readmissions Reduction Program (HRRP), established in 2012, permits Medicare to reduce payments to hospitals with excess readmissions including $\mathrm{CABG}$ surgical procedures which was added under the umbrella of the program in 2017 [13]. This program along with the continuing rise in number of aging patients with comorbid conditions undergoing cardiac surgeries will lead to increased burden on hospitals, care facilities, and payers. Therefore, it is important to develop strategies that target patient comorbidities and overcome disparities. Also, it is necessary to address avoidable perioperative complications developing as a consequence of perioperative stroke.

\section{Strengths and limitations}

This study was conducted utilizing hospital claims databases obtained from the HCUP, as they allow the analysis of a large number of patients and facilitates multi-state and multi-center analysis and comparison. There is a uniform format of collecting data in SID which made it easier to assess the possible factors contributing to this result. A major strength of this study is its large cohort size, with sufficient cases in each sex, age, and race groups to allow generalizability and population analysis. Furthermore, the usage of POA enables us to differentiate between strokes that occurred as a post-operative complication and patients presenting with strokes. This data set contains patients insured from all payer types (including the uninsured) which is necessary to investigate the effectiveness of readmissions reduction measures and is of interest to multiple parties.

Despite some of the strengths, our study should be interpreted with the following limitations in mind. First, retrospective studies done with hospital claims data do not allow us to control for stroke-specific severity measures and stroke type. Furthermore, causes of readmission are multifactorial (social, quality of care, patient, and access to care); therefore, classification of a readmission as avoidable requires profounder, prospective, circumstantial analyses. Third, in the development of complications, it was uncertain whether the complications developed as a morbidity associated with the development of perioperative stroke or vice versa. Fourth, severity for which patient underwent surgery could not be accounted for. Last, postoperative complications were obtained from ICD-9 coding information and were not validated with patient medical records. Hospital claims data are prone to errors in data reporting.

A better understanding of why stroke was statistically not proven significant in terms of 30-day readmission can be tackled through a prospective study.

\section{Conclusion}

Overall, the rate of postoperative stroke remains to be relatively high after cardiac surgeries. Patients who underwent cardiac surgeries were at an increased risk of being readmitted if they had the following characteristics: female gender, age above 65 , none white race, lower household incomes and increased comorbidities. Several perioperative complications amplified the risk of 30-day readmissions including cardiovascular, respiratory, gastrointestinal, renal and infection. However, perioperative stroke was not amongst them.

\section{Supplementary Material}

Suppl 1. Surgical Procedure CCS and ICD-9-CM Codes.

Suppl 2. ICD-9 Codes for Perioperative Complications.

\section{Acknowledgments}

Thanks to the University of Pittsburgh IRB office and the Department of Statistics.

\section{Financial Disclosure}

None to declare.

\section{Conflict of Interest}

None to declare. 


\section{Informed Consent}

Not applicable.

\section{Author Contributions}

Nada Alrifai: hypothesis, project leader, literature review, manuscript writing, editing and review; Laith Alhuneafat: data analysis, manuscript writing, editing and review, and submission; Khaled Al-Robaidi: data Analysis, manuscript editing; Samir S. Al-Ghazawi: manuscript editing; Parthasarathy D. Thirumala: corresponding author, project supervision, and review.

\section{Data Availability}

Any inquiries regarding supporting data availability of this study should be directed to the corresponding author.

\section{References}

1. English WJ. Rewarding provider performance: aligning incentives in Medicare. Ann Intern Med. 2008;148:636

2. Joynt KE, Jha AK. A path forward on Medicare readmissions. N Engl J Med. 2013;368(13):1175-1177.

3. Lawson EH, Hall BL, Louie R, Ettner SL, Zingmond DS, Han L, Rapp M, et al. Association between occurrence of a postoperative complication and readmission: implications for quality improvement and cost savings. Ann Surg. 2013;258(1):10-18.

4. Hannan EL, Zhong Y, Lahey SJ, Culliford AT, Gold JP, Smith CR, Higgins RS, et al. 30-day readmissions after coronary artery bypass graft surgery in New York State. JACC Cardiovasc Interv. 2011;4(5):569-576.

5. Hannan EL, Racz MJ, Walford G, Ryan TJ, Isom OW, Bennett E, Jones RH. Predictors of readmission for complications of coronary artery bypass graft surgery. JAMA. 2003;290(6):773-780.

6. Bucerius J, Gummert JF, Borger MA, Walther T, Doll N, Onnasch JF, Metz S, et al. Stroke after cardiac surgery: a risk factor analysis of 16,184 consecutive adult patients. Ann Thorac Surg. 2003;75(2):472-478.

7. Parikh S, Cohen JR. Perioperative stroke after general surgical procedures. N Y State J Med. 1993;93(3):162165.

8. Hart R, Hindman B. Mechanisms of perioperative cerebral infarction. Stroke. 1982;13(6):766-773.

9. Bateman BT, Schumacher HC, Wang S, Shaefi S, Berman MF. Perioperative acute ischemic stroke in noncardiac and nonvascular surgery: incidence, risk factors, and outcomes. Anesthesiology. 2009;110(2):231-238.

10. Mozaffarian D, Benjamin EJ, Go AS, Arnett DK, Blaha MJ, Cushman M, de Ferranti S, et al. Heart disease and stroke statistics-2015 update: a report from the American Heart Association. Circulation. 2015;131(4):e29-322.
11. Sharifpour M, Moore LE, Shanks AM, Didier TJ, Kheterpal S, Mashour GA. Incidence, predictors, and outcomes of perioperative stroke in noncarotid major vascular surgery. Anesth Analg. 2013;116(2):424-434.

12. Mashour GA, Shanks AM, Kheterpal S. Perioperative stroke and associated mortality after noncardiac, nonneurologic surgery. Anesthesiology. 2011;114(6):1289-1296.

13. Centers for Medicare and Medicaid Services 2019. Accessed October 11, 2019. https://www.cms.gov.

14. Barrett M, Raetzman S, Andrews R. Overview of Key Readmission Measures and Methods. 2012. HCUP Methods Series Report \#2012-04. ONLINE December 20, 2012. U.S. Agency for Healthcare Research and Quality. Available: http://www.hcupus.ahrq.gov/reports/methods/ methods.jsp.

15. Healthcare Cost and Utilization Project (HCUP) 2013, Agency for Healthcare Research and Quality. Accessed October 7, 2019. https://www.ahrq.gov/.

16. Ovbiagele B, Nguyen-Huynh MN. Stroke epidemiology: advancing our understanding of disease mechanism and therapy. Neurotherapeutics. 2011;8(3):319-329.

17. Ricotta JJ, Faggioli GL, Castilone A, Hassett JM. Risk factors for stroke after cardiac surgery: Buffalo CardiacCerebral Study Group. J Vasc Surg. 1995;21(2):359-363; discussion 364.

18. Carrascal Y, Guerrero AL, Blanco M, Valenzuela H, Pareja P, Laguna G. Postoperative stroke related to cardiac surgery in octogenarians. Interact Cardiovasc Thorac Surg. 2014;18(5):596-601.

19. Salazar JD, Wityk RJ, Grega MA, Borowicz LM, Doty JR, Petrofski JA, Baumgartner WA. Stroke after cardiac surgery: short- and long-term outcomes. Ann Thorac Surg. 2001;72(4):1195-1201; discussion 1201-1192.

20. Li Z, Armstrong EJ, Parker JP, Danielsen B, Romano PS. Hospital variation in readmission after coronary artery bypass surgery in California. Circ Cardiovasc Qual Outcomes. 2012;5(5):729-737.

21. Feng TR, White RS, Gaber-Baylis LK, Turnbull ZA, Rong LQ. Coronary artery bypass graft readmission rates and risk factors - A retrospective cohort study. Int J Surg. 2018;54(Pt A):7-17.

22. Maniar HS, Bell JM, Moon MR, Meyers BF, Marsala J, Lawton JS, Damiano RJ, Jr. Prospective evaluation of patients readmitted after cardiac surgery: analysis of outcomes and identification of risk factors. J Thorac Cardiovasc Surg. 2014;147(3):1013-1018.

23. Feng TR, Hoyler MM, Ma X, Rong LQ, White RS. Insurance status and socioeconomic markers affect readmission rates after cardiac valve surgery. J Cardiothorac Vasc Anesth. 2020;34(3):668-678.

24. Kowalewski M, Pawliszak W, Malvindi PG, Bokszanski MP, Perlinski D, Raffa GM, Kowalkowska ME, et al. Off-pump coronary artery bypass grafting improves short-term outcomes in high-risk patients compared with on-pump coronary artery bypass grafting: Meta-analysis. J Thorac Cardiovasc Surg. 2016;151(1):60-77 e61-58.

25. BhaskerRao B, VanHimbergen D, Edmonds HL, Jr., Jaber S, Ali AT, Pagni S, Koenig S, et al. Evidence for improved cerebral function after minimally invasive bypass sur- 
gery. J Card Surg. 1998;13(1):27-31.

26. Iqbal J, Ghaffar A, Shahbaz A, Abid AR. Stroke after coronary artery bypass surgery with and without cardiopulmonary bypass. J Ayub Med Coll Abbottabad. 2014; 26(2):123-128.

27. Moody DM, Bell MA, Challa VR, Johnston WE, Prough DS. Brain microemboli during cardiac surgery or aortography. Ann Neurol. 1990;28(4):477-486.

28. Li Z, Amsterdam EA, Danielsen B, Hoegh H, Young JN, Armstrong EJ. Intraoperative conversion from off-pump to on-pump coronary artery bypass is associated with increased 30-day hospital readmission. Ann Thorac Surg. 2014;98(1):16-22.

29. Espinoza J, Camporrontondo M, Vrancic M, et al. 30-day readmission score after cardiac surgery. Clin Trials Regul Sci Cardiol. 2016;20:1-5.

30. Iribarne A, Chang H, Alexander JH, Gillinov AM, Moquete E, Puskas JD, Bagiella E, et al. Readmissions after cardiac surgery: experience of the National Institutes of Health/Canadian Institutes of Health research cardiothoracic surgical trials network. Ann Thorac Surg. 2014;98(4):1274-1280.

31. Foley N, Teasell R, Salter K, Kruger E, Martino R. Dysphagia treatment post stroke: a systematic review of ran- domised controlled trials. Age Ageing. 2008;37(3):258264.

32. Stott DJ, Falconer A, Miller H, Tilston JC, Langhorne P. Urinary tract infection after stroke. QJM. 2009;102(4): 243-249.

33. Poisson SN, Johnston SC, Josephson SA. Urinary tract infections complicating stroke: mechanisms, consequences, and possible solutions. Stroke. 2010;41(4):e180-184.

34. Ersoz M, Ulusoy H, Oktar MA, Akyuz M. Urinary tract infection and bacteriurua in stroke patients: frequencies, pathogen microorganisms, and risk factors. Am J Phys Med Rehabil. 2007;86(9):734-741.

35. Bravata DM, Ho SY, Meehan TP, Brass LM, Concato J. Readmission and death after hospitalization for acute ischemic stroke: 5-year follow-up in the medicare population. Stroke. 2007;38(6):1899-1904.

36. Rao A, Barrow E, Vuik S, Darzi A, Aylin P. Systematic review of hospital readmissions in stroke patients. Stroke Res Treat. 2016;2016:9325368.

37. Wheatley B, DeJong G, Sutton JP. Managed care and the transformation of the medical rehabilitation industry. Health Care Manage Rev. 1997;22(3):25-39.

38. Forgeson G. Guidelines of the use of expensive medicines. N Z Med J. 1995;108(996):111. 